\title{
Projective embeddings of dual polar spaces arising from a class of alternative division rings
}

\author{
Bart De Bruyn ${ }^{1}$ \\ Department of mathematics \\ Ghent University \\ Gent, Belgium \\ Hendrik Van Maldeghem ${ }^{2}$ \\ Department of Mathematics \\ Ghent University \\ Gent, Belgium
}

\begin{abstract}
We discuss some recent results of us regarding a class of polar spaces which includes the nonembeddable polar spaces introduced by Tits [3]. These results include an elementary construction of the polar space, a construction of a polarized embedding of the corresponding dual polar space and the determination whether this projective embedding is universal and unique (as a polarized embedding).

Keywords: nonembeddable polar space, Cayley-Dickson division algebra, alternative division ring, dual polar space, (polarized, universal) projective embedding
\end{abstract}

\begin{tabular}{lll}
\hline 1 & Email: bdb@cage.ugent.be \\
2 & Email: hvm@cage.ugent.be
\end{tabular} 


\section{Introduction}

A polar space of rank $n \geq 1([3])$ is a pair $(P, \mathcal{S})$ consisting of a nonempty set $P$, whose elements are called points, and a collection $\mathcal{S}$ of subsets of $P$, called subspaces, satisfying the following four properties:

- Every subspace, together with the subspaces contained in it, defines a projective space of dimension at most $n-1$.

- The intersection of two subspaces is again a subspace.

- If $M$ is a subspace of dimension $n-1$, then through every point $x$ not contained in $M$, there is a unique subspace $M^{\prime}$ of dimension $n-1$ intersecting $M$ in a subspace of dimension $n-2$. Moreover, $M \cap M^{\prime}$ consists of all points of $M$ that are contained in a 1-dimensional subspace together with $x$.

- There exist two disjoint subspaces of dimension $n-1$.

A polar space is called thick if every line (i.e. one-dimensional subspace) contains at least three points and if every $(n-2)$-dimensional subspace is contained in at least three $(n-1)$-dimensional subspaces. The polar spaces of rank 2 are the so-called generalized quadrangles. Tits [3] classified all thick polar spaces of rank at least three. He found that with exception of one class, all such polar spaces are fully embeddable in a projective space and arise from a pseudo-quadratic form on a vector space. The exceptional nonembeddable polar spaces only exist for rank 3 and are obtained from algebraic simple groups of type $E_{7}$. There exists a nonembeddable polar space for each Cayley-Dickson division algebra. In [1], we realized the following goals.

- We gave an elementary construction of any nonembeddable polar space.

- We used this to construct a full projective embedding of the corresponding dual polar space. A construction of a full embedding of this dual polar space is also implicit in the work of Mühlherr [2].

- We proved several new results about this projective embedding.

In the present note, we discuss these results. The methods to achieve the above goals do not only work for the nonembeddable polar spaces, but for all members of a family of polar spaces derived from certain alternative division rings. We are going to present our results in this more general context.

\section{Alternative division rings}

An alternative division ring is a set $\mathbb{D}$ of size at least 2 which is endowed with two binary operations, an addition + and a multiplication $\cdot$, satisfying the 
following properties:

- $(\mathbb{D},+, \cdot)$ is a nonassociative (= not necessarily associative) ring with identity element 1 for the multiplication;

- if 0 denotes the neutral element for the addition, then for every $a \in \mathbb{D} \backslash\{0\}$, there exists a (necessarily unique) element $a^{-1} \in \mathbb{D}$ such that $a^{-1} \cdot a=1=$ $a \cdot a^{-1}$

- for every $a \in \mathbb{D} \backslash\{0\}$ and every $b \in \mathbb{D}$, we have $a^{-1} \cdot(a \cdot b)=b=(b \cdot a) \cdot a^{-1}$.

The center $Z(\mathbb{D})$ of $\mathbb{D}$ consists of all $a \in \mathbb{D}$ such that $a b=b a, a(b c)=(a b) c$, $(b a) c=b(a c)$ and $(b c) a=b(c a)$ for all $b, c \in \mathbb{D}$. The center $Z(\mathbb{D})$ is a field.

Suppose $\mathbb{F}$ is a subfield of $Z(\mathbb{D})$. We say that $\mathbb{D}$ is quadratic over $\mathbb{F}$ if there exist (necessarily unique) functions $T: \mathbb{D} \rightarrow \mathbb{F}$ and $N: \mathbb{D} \rightarrow \mathbb{F}$ such that:

- $a^{2}-T(a) a+N(a)=0$ for any $a \in \mathbb{D}$;

- $T(a)=2 a$ and $N(a)=a^{2}$ for any $a \in \mathbb{F}$.

The following proposition is precisely Theorem 20.3 of Tits and Weiss [4].

Proposition 2.1 ([4]) Exactly one of the following holds:

(a) $\mathbb{D}$ is a field and $\mathbb{F}=\mathbb{D}$;

(b) $\mathbb{D}$ is a field and is a separable quadratic extension of $\mathbb{F}$;

(c) $\mathbb{D}$ is a field of characteristic 2 and $\mathbb{D}^{2} \subseteq \mathbb{F} \neq \mathbb{D}$;

$(d) \mathbb{D}$ is a quaternion division algebra and $\mathbb{F}=Z(\mathbb{D})$;

(e) $\mathbb{D}$ is a Cayley-Dickson division algebra and $\mathbb{F}=Z(\mathbb{D})$.

If we put $a^{\sigma}:=T(a)-a$ for every $a \in \mathbb{D}$, then $\sigma$ is an involution of $\mathbb{D}$ and $N(a)=a^{\sigma} a$ for every $a \in \mathbb{D}$. The involution $\sigma$ is trivial if case $(a)$ or $(c)$ occurs. In case (b), $\sigma$ is the unique nontrivial element of the Galois group $\operatorname{Gal}(\mathbb{D} / \mathbb{F})$. In cases $(d)$ and $(e), \sigma$ is the standard involution of $\mathbb{D}$.

\section{Construction of the polar space}

With every pair $\mathcal{T}=(\mathbb{D}, \mathbb{F})$ as above, a polar space $\mathcal{P}_{\mathcal{T}}$ of rank 3 can be constructed. There are six types of points, labeled as follows:

$$
(\infty), \quad\left(x_{1}\right), \quad\left(x_{1}, x_{2}\right), \quad\left(x_{1}, x_{2} ; k\right), \quad\left(x_{1}, x_{2}, x_{3} ; k\right), \quad\left(x_{1}, x_{2}, x_{3}, x_{4} ; k\right)
$$

where $x_{1}, x_{2}, x_{3}, x_{4} \in \mathbb{D}$ and $k \in \mathbb{F}$. In [1], we defined twelve sets of points which we called lines and eight sets of points which we called planes. These sets of points are indexed by certain parameters which satisfy certain restrictions. 
The planes can be labeled as

$$
\begin{gathered}
{[\infty],[k],\left[x_{1} ; k\right],\left[x_{1} ; k, \ell\right],\left[x_{1}, x_{2} ; k\right],\left[x_{1}, x_{2} ; k, \ell\right],} \\
{\left[x_{1}, x_{2}, x_{3} ; k, \ell\right],\left[x_{1}, x_{2}, x_{3} ; k, \ell, m\right]}
\end{gathered}
$$

where $x_{1}, x_{2}, x_{3} \in \mathbb{D}$ and $k, \ell, m \in \mathbb{F}$. The lines can be labeled as:

- $L_{1}$;

- $L_{2}(x)$ where $x \in \mathbb{D}$;

- $L_{3}(x, k)$ where $x \in \mathbb{D}$ and $k \in \mathbb{F}$;

- $L_{4}(x, y, k)$ where $x, y \in \mathbb{D}$ and $k \in \mathbb{F}$;

- $L_{5}(x, y, z)$ where $x, y, z \in \mathbb{D}$;

- $L_{6}(x, y, z, k)$ where $x, y, z \in \mathbb{D}$ and $k \in \mathbb{F}$;

- $L_{7}(x, y, z, u, k)$ where $x, y, z, u \in \mathbb{D}$ and $k \in \mathbb{F}$ such that $x=-y^{\sigma}$;

- $L_{8}(x, y, u, v, w, k)$ where $x, y, u, v, w \in \mathbb{D}$ and $k \in \mathbb{F}$ such that $u=-x^{\sigma}$;

- $L_{9}(x, y, z, u, v, w, k)$ where $x, y, z, u, v, w \in \mathbb{D}$ and $k \in \mathbb{F}$ such that $y=$ $-u^{\sigma}-z^{\sigma} x$

- $L_{10}\left(x, y, u, v, w, k_{1}, k_{2}\right)$ where $x, y, u, v, w \in \mathbb{D}$ and $k_{1}, k_{2} \in \mathbb{F}$ such that $v=x^{\sigma}+k_{1} u$

- $L_{11}\left(x, y, z, u, v, w, k_{1}, k_{2}\right)$ where $x, y, z, u, v, w \in \mathbb{D}$ and $k_{1}, k_{2} \in \mathbb{F}$ such that $v=x^{\sigma} z+y^{\sigma}+k_{1} u$;

- $L_{12}\left(x, y, z, u, v, w, r, k_{1}, k_{2}\right)$ where $x, y, z, u, v, w, r \in \mathbb{D}$ and $k_{1}, k_{2} \in \mathbb{F}$ such that $r=z^{\sigma}-y^{\sigma}(x u-v)+k_{1} u-x^{\sigma} w$.

The following was proved in [1].

Theorem 3.1 The points, lines and planes determine a polar space $\mathcal{P}_{\mathcal{T}}$ of rank 3.

If case (a) of Proposition 2.1 occurs, then $\mathcal{P}_{\mathcal{T}}$ is isomorphic to the symplectic polar space $W(5, \mathbb{F})$ defined by a symplectic polarity of $\mathrm{PG}(5, \mathbb{F})$. If case (b) of Proposition 2.1 occurs, then $\mathcal{P}_{\mathcal{T}}$ is isomorphic to the Hermitian polar space $H(5, \mathbb{D}, \sigma)$ defined by a $\sigma$-Hermitian polarity of $\operatorname{PG}(5, \mathbb{D})$. In case $(\mathrm{c})$, $\mathcal{P}_{\mathcal{T}}$ is a so-called polar space of mixed type and in case $(\mathrm{d}), \mathcal{P}_{\mathcal{T}}$ is called a quaternionic polar space. If case (e) of Proposition 2.1 occurs, then $\mathcal{P}_{\mathcal{T}}$ is the nonembeddable polar space for which $\mathbb{D}$ is the corresponding Cayley-Dickson division algebra. 


\section{Projective embedding of the corresponding dual po- lar space}

Let $\Delta_{\mathcal{T}}$ denote the dual polar space associated with $\mathcal{P}_{\mathcal{T}}$. So, $\Delta_{\mathcal{T}}$ is the pointline geometry whose points are the planes of $\mathcal{P}_{\mathcal{T}}$ and whose lines are the lines of $\mathcal{P}_{\mathcal{T}}$, with incidence being reverse containment. A full projective embedding of $\Delta_{\mathcal{T}}$ is called absolutely universal when every other embedding can by obtained from it by means of a so-called projection. A full projective embedding $e^{\prime}$ of $\Delta_{\mathcal{T}}$ into a projective space $\Sigma$ is called polarized if for every every point $x$ of $\Delta_{\mathcal{T}}$ there exists a (necessarily unique) hyperplane $\Pi_{x}$ of $\Sigma$ through $e^{\prime}(x)$ containing all points $e^{\prime}(y)$ where $y$ is some point of $\Delta_{\mathcal{T}}$ at distance at most 2 from $x$ (in the collinearity graph).

The alternative division ring $\mathbb{D}$ can be regarded as an $n$-dimensional vector space over $\mathbb{F}$ and hence

$$
V:=\mathbb{F} \times \mathbb{F} \times \mathbb{F} \times \mathbb{F} \times \mathbb{D} \times \mathbb{D} \times \mathbb{D} \times \mathbb{F} \times \mathbb{F} \times \mathbb{F} \times \mathbb{D} \times \mathbb{D} \times \mathbb{D} \times \mathbb{F}
$$

can be regarded as an $(8+6 n)$-dimensional vector space over $\mathbb{F}$. In [1], we constructed a certain map $e$ from the point-set of $\Delta_{\mathcal{T}}$ (i.e. the set of planes of $\left.\mathcal{P}_{\mathcal{T}}\right)$ to the set of points of $\mathrm{PG}(V)$ and showed the following.

Theorem 4.1 - The map e defines a full polarized embedding of $\Delta_{\mathcal{T}}$ into $\mathrm{PG}(V)$.

- If $\Delta_{\mathcal{T}}$ is a symplectic or Hermitian dual polar space, then e is isomorphic to the so-called Grassmann embedding of $\Delta_{\mathcal{T}}$.

- If $|\mathbb{F}| \neq 2$, then $e$ is absolutely universal.

- $e$ is the unique polarized embedding of $\Delta_{\mathcal{T}}$ if and only if $|\mathbb{F}| \neq 2$ and either $\operatorname{char}(\mathbb{F}) \neq 2$ or $\sigma$ nontrivial.

\section{References}

[1] De Bruyn, B., and H. Van Maldeghem, Polar spaces of rank 3 defined over quadratic composition division algebras, Preprint, 2012.

[2] Mühlherr, B., A geometric approach to non-embeddable polar spaces of rank 3, Bull. Soc. Math. Belg. Sér. A 42 (1990), 577-594.

[3] Tits, J., "Buildings of spherical type and finite BN-pairs," Lecture Notes in Mathematics 386, Springer-Verlag, Berlin-New York, 1974.

[4] Tits, J., and R. M. Weiss, "Moufang polygons," Springer Monographs in Mathematics, Springer-Verlag, Berlin, 2002. 